\title{
Synergistic effects of unripe raspberry extracts (Rubus chingii) and antibiotics against three bacteria
}

\author{
Huichuan JIANG ${ }^{1 *}$, Jing YANG ${ }^{2 \# \star}$ (D), Yanling $\mathrm{FAN}^{2}$, Yongping $\mathrm{LIU}^{2}$
}

\begin{abstract}
Dried unripe raspberries (Rubus chingii), a kind of Chinese herbal medicine, have been used for many years in China. They were prepared as unpurified raspberry extract (URE) by ethanol extraction, and purified raspberry extract (PRE) by macroporous resin. Synergistic effects between the URE (or the PRE) and ampicillin, erythromycin, tetracycline or streptomycin against Escherichia coli, Staphylococcus aureus and Salmonella enterica were carried out using checkerboard microdilution and time-kill curves, respectively. The results showed that all pairwise combinations revealed synergistic effects, with $0.10 \leq$ fractional inhibitory concentration index $\leq 0.50$ and more than $2 \times \log _{10}$ reduction in colony-forming units $/ \mathrm{mL}$. In particular, the erythromycin concentration in the combinations was reduced to 1/50-1/100 of its dose alone while retaining its effectiveness against $S$. aureus and E. coli. The synergistic effects of raspberry extracts and four antibiotics against three bacteria were related to the abundance of polyphenols in the extracts, especially ellagic acid. The extracts may be a dietary supplement and/or natural antimicrobials.
\end{abstract}

Keywords: antibiotics; ellagic acid; natural antimicrobials; raspberry extracts; synergistic effects.

Practical Application: Raspberry extracts may be a dietary supplement and/or natural antimicrobials.

\section{Introduction}

The unripe (green) raspberry of Rubus chingii Hu, containing high medicinal ingredients e.g. polyphenols, terpenoids, saponins, polysaccharides, alkaloids, and essential oils, exhibits antioxidant, anti-bacteria, anti-complement and anticancer activities (Yang et al., 2019; Yu et al., 2019). It is the only plant of Rubus species included in The Chinese Pharmacopoeia and used to improve kidney functions, such as enuresis and urinary frequency, in Traditional Chinese Medicine for many years (Chinese Pharmacopoeia Commission, 2015). Whereas, the ripe fruits of $R$. chingii, R. idaeus, R. occidental and Rubus spp., called "fu-pen-zi" in Chinese, or raspberry in English, mainly contain tannin and anthocyanins for daily dietary (Krauze-Baranowska et al., 2014; Xiao et al., 2017; Yu et al., 2019).

The widespread uses of antibiotics in healthcare and agriculture have been beneficial, but have also had unexpected negative consequences on human beings and the environment. The excessive use of antibiotics creates a new pollutant that has been released into rivers and soil through exposure to human waste, which disturbed the entire biosphere (Gillings, 2013; Popowska et al., 2012). The evolution of pathogenic microorganisms has been increased in the environment due to high concentrations of antibiotics. The adaption time require for Escherichia coli to go from zero to 1,000 -fold the minimal inhibitory concentration (MIC) in a gradient medium was just $10 \mathrm{~d}$ when expose to trimethoprim or $12 \mathrm{~d}$ to ciprofloxacin (Baym et al., 2016a; Belica et al., 2017). Increasing antibiotic doses and the use of new synthetic antibiotics not only do not solve the problem of antibiotic-resistant bacteria, but also cause more serious ecological pollution. Fortunately, plant evolution involves the struggle between plants and pathogenic microorganisms or herbivores (Baym et al., 2016a; Gillings, 2013). In this process, plants evolve thousands of metabolites, such as flavonoids and non-flavonoids polyphenols, to defend against biotic and abiotic stresses (Zhang et al., 2010). Therefore, Newman found that $\sim 50 \%$ of new medicines developed from 1981 to 2006 were directly or indirectly derived from plant products (Newman \& Cragg, 2007). The most representative of the plant-derived drugs is veregen, a mainly green tea extract that includes a variety of polyphenols. It was the first Chinese herbal medicine approved by the US Food and Drug Administration (Schmidt et al., 2007). The Realgar-Indigo naturalis formula (RIF) based on 100,000 Traditional Chinese Medicine formulae is very effective in treating human acute promyelocytic leukemia. RIF contains extracts of two plants (Indigo naturalis and Salvia miltiorrhiza) as well as minerals (Wang et al., 2008). Extracts of freeze-dried black raspberries (R. occidentalis) may be considered as potential sources for polyphenolic drugs in the treatment of diabetes mellitus (Xiao et al., 2017). Our previous study on four $R$. Chingii extracts found that ellagic acid, brevifolin carboxylic acid and kaempferol-3-rutinoside were the highest proportion of 11 tested phenolic compounds. Their MICs against Escherichia coli, Staphylococcus aureus and Salmonella enterica ranged from 2-20 mg/mL (Yang et al., 2019).

${ }^{1}$ Rubber Research Institute, Chinese Academy of Tropical Agricultural Sciences / Key Laboratory of Biology and Genetic Resources of Rubber Tree, Ministry of Agriculture and Rural Affairs, Haikou, China

${ }^{2}$ School of Chemical Engineering and Technology, North University of China, Taiyuan, China

*These authors are co-first author.

*Corresponding author: yangjing5152@163.com 
In addition, catechin, epicatechin, quercetin, kaempferol and isorhamnetin derived from almond skin combine with vitamins $C$ and/or E have synergistic effects to protect low density lipoprotein against oxidation in human and hamster (Chen et al., 2005). A synergistic activity is also observed when ceftazidime is combined with either apigenin or naringenin using the checkerboard method with a fractional inhibitory concentration index (FICI) between 0.01 and 0.27 against ceftazidime-resistant Enterobacter cloacae strains, whereas ceftazidime plus quercetin has no synergy, with an FICI > 1.0 (Eumkeb \& Chukrathok, 2013). Similarly, green tea extract (containing $47.92 \%$ epigallocatechin-3-gallate) or just epigallocatechin-3-gallate in combination with antibiotics (metronidazole or tetracycline) shows synergistic activities against Porphyromonas gingivalis, a key pathogen in the chronic form of periodontitis (Fournier-Larente et al., 2016). Fruit extract ( $R$. chingii) combined with fluconazole, has an antifungal activity against fluconazole-resistant Candida albicans (a diploid fungus) (Han et al., 2016). Moreover, the mixture of raspberry and adzuki bean more effectively attenuate H9c2 cardiomycytes under oxidative stress than the individual extracts (Wang et al., 2012). Here, the synergistic effects of multi-drugs, especially antibiotics and plant-derived antibacterials, may compensate for the deficiencies of the drugs themselves and address more complex diseases that have multi-causal etiology and complex pathogen physiology (Baym et al., 2016b; Gertsch, 2011; Yang et al., 2014).

The purpose of this study was to provide a basic for the synergistic effects of raspberry extract and antibiotics. Therefore, the synergistic effects between two unripe raspberry extracts (R. chingii) and four antibiotics against bacteria were evaluated using checkerboard microdilution and time-kill curves. Firstly, unripe raspberry extracts (URE) by ethanol extraction, and purified raspberry extract (PRE) by macroporous resin were prepared according to Yang et al.(2019). Secondly, the MICs of two extracts and four antibiotics against E. coli, S. aureus and $S$. enterica were investigated respectively. Then, pairwise combinations of extracts (URE or PRE) and antibiotics (ampicillin, erythromycin, tetracycline or streptomycin) against three bacteria were independently assayed.

\section{Materials and Methods}

\subsection{Reagents}

Four antibiotics including ampicillin (AMP), erythromycin (ERY), tetracycline (TET), streptomycin (STR) (Nanjing Oddfoni Biological Technology Co., Ltd. China) and XDA-6 macroporous resins (Lanxiao Technology New Material Co., Ltd. China) were used in this study. The unripe raspberry of $R$. chingii $\mathrm{Hu}$ was harvested and dried in Zhejiang $\left(\mathrm{N} 30^{\circ}, \mathrm{E} 120^{\circ}\right)$, by Hebei Hanyaocaotang Pharmacy Co. Ltd. China). The powder of dried unripe raspberries was purchased from local pharmacy (Taiyuan, China).

\subsection{Bacteria and culture conditions}

China General Microbiological Culture Collection Center (CGMCC) provided Escherichia coli (CGMCC1.12883), Staphylococcus aureus (CGMCC1.282), and Salmonella enterica (CGMCC1.755). They were incubated with shaking (200 rpm) in $\mathrm{LB}$ medium at $37^{\circ} \mathrm{C}$ incubator.

\subsection{Extraction and purification}

The extracts were prepared according to Yang et al.(2019). Briefly, dried unripe raspberry powder was extracted using the ultrasonic-assisted ethanol method, in which $10 \mathrm{mg} / \mathrm{mL}$ of powder was extracted in $52 \%$ ethanol at $360 \mathrm{~W}$ for $45 \mathrm{~min}$, twice, using a JOYN-15AL Ultrasonic apparatus (Shanghaiqiaoyue Co.). One part of concentrated extraction solution was freeze-dried into a brown powder, as unpurified raspberry extract (URE). The rest concentrated solution was purified by XDA- 6 macroporous resin under the following purification conditions: six bed volumes (BVs)/h as the velocity of adsorption, $60 \%$ ethanol as the elution reagent, with four $\mathrm{BV} / \mathrm{h}$ as the elution velocity and five $\mathrm{BV}$ effluent. The concentrated purified solution was freeze-dried into a light brown powder, as purified raspberry extract (PRE).

\subsection{MIC and FICI}

MICs of URE or PRE and four antibiotics against the three bacteria were determined by the checkerboard microdilution, respectively (Hall et al., 1983). The extract stock solutions were freshly prepared by dissolving 64 or $80 \mathrm{mg}$ of the URE or PRE powder in $1 \%$ of DMSO solution filtered through a $0.22 \mu \mathrm{m}$ filter membrane. Antibiotics were dissolved diluted in sterilized deionized water according to different concentration requirement. All samples bacteria suspensions were diluted in fresh $\mathrm{LB}$ to adjust to an $\mathrm{OD}_{660}$ of 0.2 . Equal volumes $(100 \mu \mathrm{L})$ of bacteria and serial two-fold dilutions of extract solution or antibiotic solution in LB medium were mixed into the wells of a 96-well microplate. Wells with no bacteria or no compounds were negative control. After an incubation with shaking (50 rpm) of $18-24 \mathrm{~h}$ at $37^{\circ} \mathrm{C}$ incubator, bacterial growth was recorded visually. MICs were determined as the minimum concentrations at which no bacterial growth observed.

URE or PRE was serially diluted along the ordinate of a 96-well microplate, while the antibiotics were serially diluted along the abscissa. Bacterial suspensions and MICs alone were the same as above. The FICI was calculated using the following equation: $\mathrm{FICI}=\mathrm{FIC}_{\mathrm{A}}+\mathrm{FIC}_{\mathrm{B}}=\left(\mathrm{MIC}_{\text {extract }}\right.$ in combination/ $\mathrm{MIC}_{\text {extract }}$ alone $)+\left(\mathrm{MIC}_{\text {antibiotic }}\right.$ in combination/ $\mathrm{MIC}_{\text {antibiotic }}$ alone $)$. The synergy type was judged by FICIs: $0<$ FICI $\leq 0.5$, synergy effect, S; $0.5<$ FICI $<4$, no interaction, N; and FICI $>4$, antagonism (Hall et al., 1983; Odds, 2003).

\subsection{Time-kill curve assay}

Time-kill curve assays were performed to evaluate the killing dynamics of samples and to determine the synergistic effect of URE or PRE and antibiotics combinations according to the National Committee for Clinical Laboratory Standards guidelines (National Committee for Clinical Laboratory Standards, 1999). Exponentially growing bacteria were diluted to $\sim 1 \times 10^{6}$ colony-forming units $(\mathrm{CFU}) / \mathrm{mL}$ in medium. Tubes containing $10 \mathrm{~mL}$ cultures were exposed to URE or PRE alone at $0.5 \times \mathrm{MIC}$, or antibiotic alone at $0.5 \times \mathrm{MIC}$, or URE or PRE plus antibiotics at $0.5 \times \mathrm{MIC}$ and incubated at $37^{\circ} \mathrm{C}$ incubator. One tube containing $10 \mathrm{~mL}$ culture without samples was used as a growth control. At 0, 4, 8, 12 and 24h, $20 \mu \mathrm{L}$ aliquots obtained from tubes were inoculated on agar plates for colony counts after 10 -fold serial dilution. The number 
of viable colonies was counted only from the plates containing between 30 and 300 colonies to calculate CFU/mL. Synergy was defined as a decrease of $\geq 2-\log _{10} \mathrm{CFU} / \mathrm{mL}$ induced by the drug combination compared with that by the drug alone in 24 hours, while no interaction was defined as a change of $<2-\log _{10} \mathrm{CFU} /$ mL (Wei et al., 2016).

\subsection{Statistical analysis}

All experiments were performed in triplicate. The data were expressed as the means \pm standard deviation. All statistical analyses were performed with SPSS software (Statistical Package for Social Sciences, USA, version 19.0). Statistical significance between two groups was determined using Student's t-test. Statistical significance of differences was taken as $p<0.05$.

\section{Results and discussion}

One Gram-positive bacterium ( $S$. aureus) and two Gram-negative bacteria (E. coli and S. enterica), are three common pathogens causing infections in humans. Reducing the amount of antibiotics against them is beneficial to reducing the production of multidrug-resistant superbacteria (Baym et al., 2016a, b). AMP, ERY, TET and STR with different molecular structures are the most representative broad-spectrum antibiotics. AMP inhibits and kills bacteria mainly by inhibiting their cell wall growth. The other three mainly act as protein synthesis inhibitor (Popowska et al., 2012).

In this study, the MICs of URE, PRE and four antibiotics against the three bacteria, E.coli, S. aureus and S. enterica, were summarized in Table 1 . The MICs of URE and PRE were $>4 \mathrm{mg} / \mathrm{mL}$, and the dose of PRE was half that of URE, indicating that the antibacterial activity of the purified extract was superior compared to the unpurified extract for these selected bacteria. The MICs of AMP and TET ranged from 4 to $125 \mu \mathrm{g} / \mathrm{mL}$, and those of ERY and STR ranged from 125 to $500 \mu \mathrm{g} / \mathrm{mL}$ (Table 1). Pairwise combinations of URE or PRE and the antibiotics (AMP, ERY, TET or STR) were independently assayed against E. coli, S. aureus and S.enterica. $\mathrm{FICI}_{\mathrm{URE} / \mathrm{ERY}}$ and $\mathrm{FICI}_{\mathrm{PRE} / \mathrm{ERY}}$ against $S$. aureus and $E$. coli ranged from 0.1 to 0.25 ,

Table 1. Synergistic effects of raspberry extracts and antibiotics against bacteria.

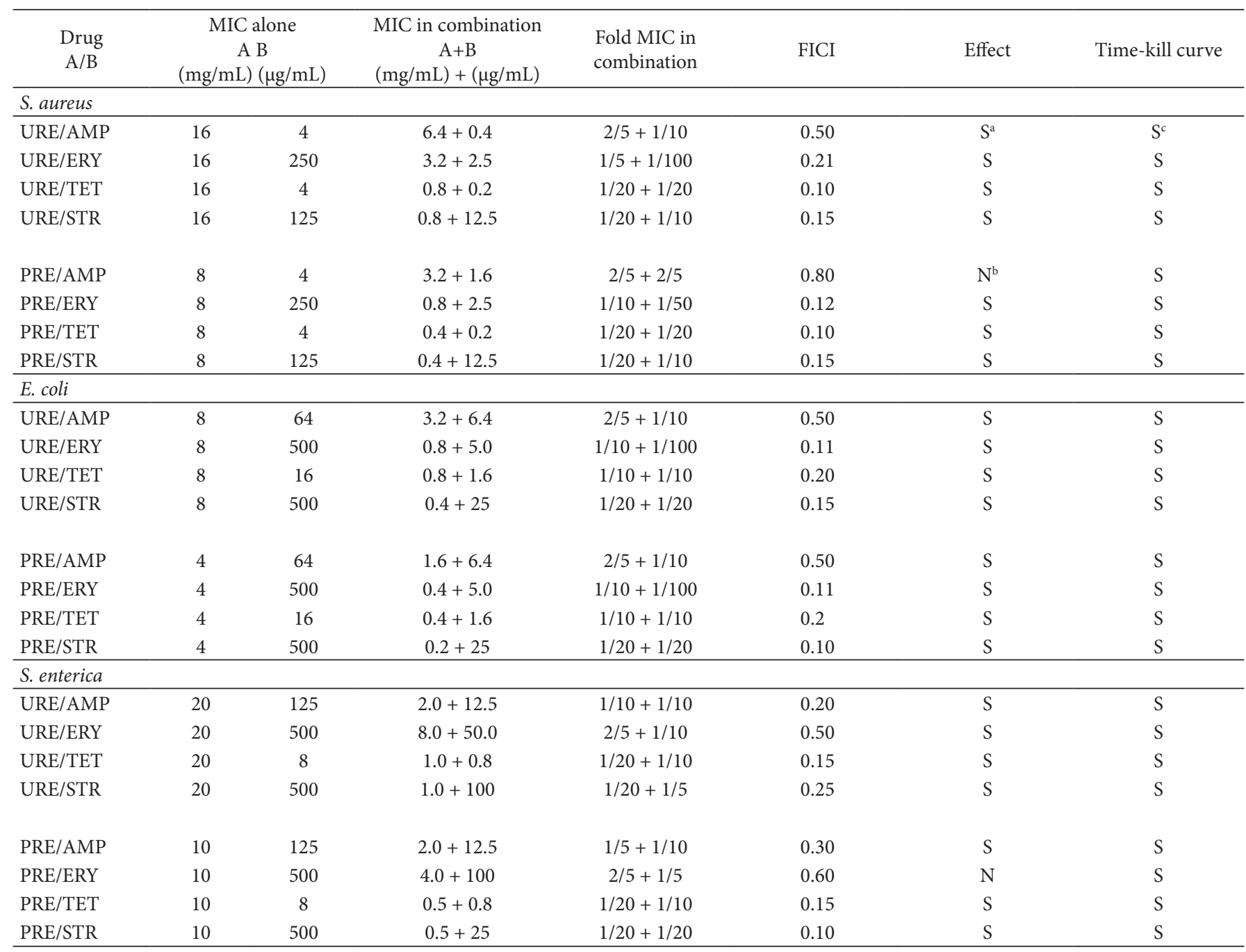

MIC, Minimal inhibitory concentration; CFU, colony forming units; AMP, ampicillin; TET, tetracycline; ERY, erythromycin; STR, streptomycin; URE, unpurified raspberry extract; PRE, purified raspberry extract; FICI $=$ FIC $_{\mathrm{A}}+\mathrm{FIC}_{\mathrm{B}}=\left(\mathrm{MIC}_{\mathrm{A}}\right.$ in combination/ $\mathrm{MIC}_{\mathrm{A}}$ alone $)+\left(\mathrm{MIC}_{\mathrm{B}}\right.$ in combination/ MIC ${ }_{\mathrm{B}}$ alone $)$; ${ }^{\text {S }}$, synergy: $0<\mathrm{FICI} \leq 0.5$; ${ }^{\mathrm{b}} \mathrm{N}$, no interaction: $0.5<$ FICI $<4$; ${ }^{c} S$, synergy with a decrease of $\geq 2 \times \log _{10}$ CFU $/ \mathrm{mL}$ by Figure 1 . 
which indicated significant synergistic effects $($ FICI $\leq 0.5)$. The ERY concentration in the combinations was reduced to $1 / 50-1 / 100$ of its normal independent dose while retaining its effectiveness against $S$. aureus and E. coli. However, FICI $_{\mathrm{URE} / \mathrm{ERY}}$ and FICI $_{\mathrm{PRE} / \mathrm{ERY}}$ against S.enterica were 0.5 and 0.6 , respectively, which indicated almost no interaction $(0.5<$ FICI $<4.0)$. In addition, FICI $_{\text {URE/AMP }}$ and FICI $\mathrm{PRE}_{\mathrm{AMP}}$ against S.enterica were 0.2 and 0.3 , respectively, which indicated synergistic effects, while the same combinations against S.aureus and E. coli ranged from 0.5 to 0.8 , which indicated no interaction. Even so, compared with the MIC of the antibiotics alone, their levels in the combinations were markedly reduced.

To validate the synergistic effects of URE or PRE and antibiotic combinations against the three bacteria, the combinations were assessed using time-kill curve assays again. Either extract or antibiotic alone $(0.5 \times$ MIC) decreased the rate of bacterial growth compared with the control (without sample), while when the URE or PRE and any antibiotic were combined at
$0.5 \times$ MIC each, all three tested strains showed slower growth rates. There was a $>2 \times \log _{10}$ decrease in CFU $/ \mathrm{mL}$ at $24 \mathrm{~h}$ compared with URE, PRE or antibiotic alone (Figure 1). Thus, a synergistic effect was observed in all combinations, including $0<$ FICI $<1.0$. The macrolide antibiotics, clarithromycin, roxithromycin (ROX), azithromycin and spiramycin, were used to repeat the above experiments (Table S1 of Supplementary Material) based on the important role of ERY (a macrolide antibiotic) in the above result (Table 1). The time-kill curve assays of the combinations $\left(\mathrm{FICI}_{\mathrm{URE} / \mathrm{ROX}}=1.01\right.$ and $\left.\mathrm{FICI}_{\mathrm{PRE} / \mathrm{ROX}}=1.01\right)$ revealed no interactionas indicated by a $<2 \times \log _{10}$ decrease in $\mathrm{CFU} / \mathrm{mL}$ at $24 \mathrm{~h}$ compared with URE, PRE or antibiotic alone. Other combinations $(0<\mathrm{FICI}<1.0)$ showed synergy based on the time-kill curve assays. Therefore, we hypothesized that synergistic effect should occur in all cases $(0<\mathrm{FICI}<1.0)$. In addition, the PRE produced by macroporous resin purification effectively gathered the antibacterial materials, e.g. flavonoids and polyphenols, and reduced the reagent doses of combinations, which benefits their preservation, transportation and reutilization.

\section{S. aureus}
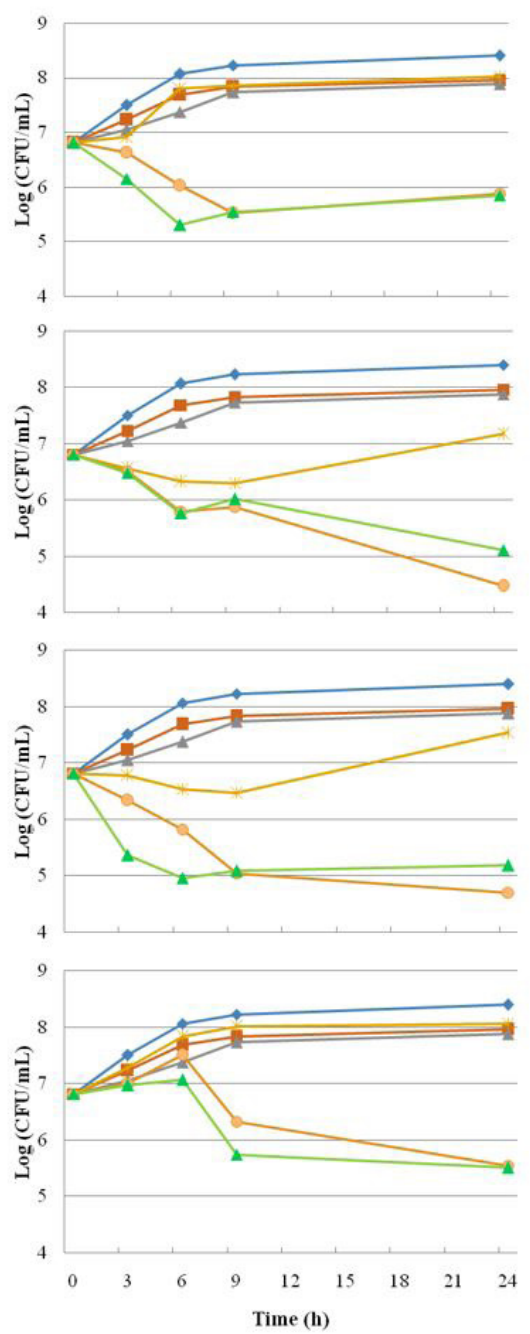

E. coli
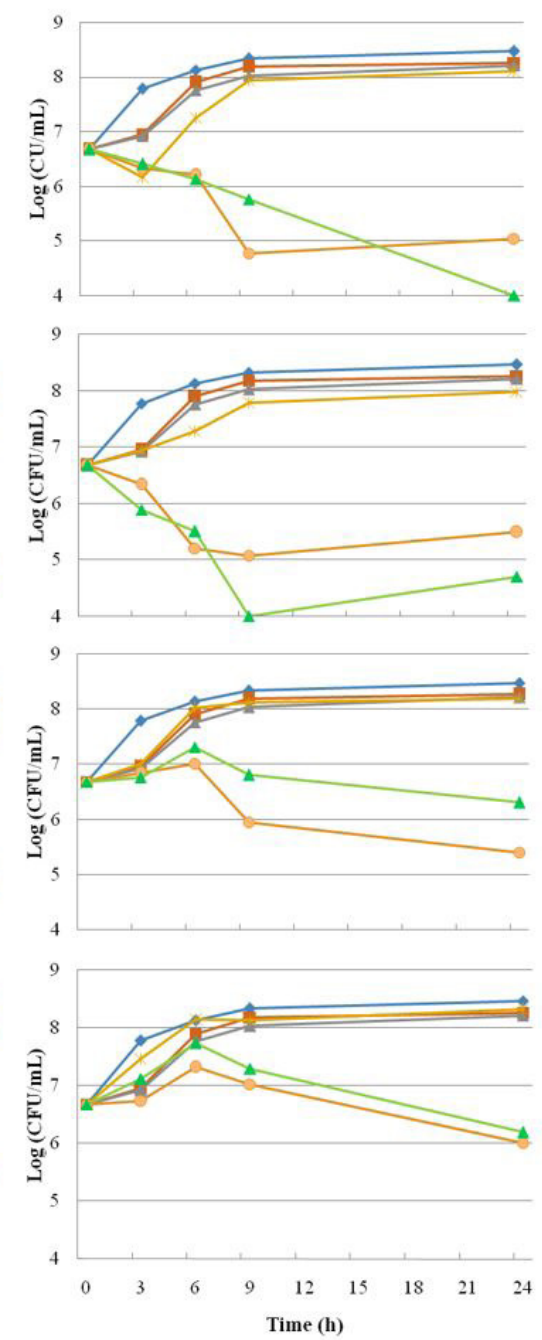

S. enterica

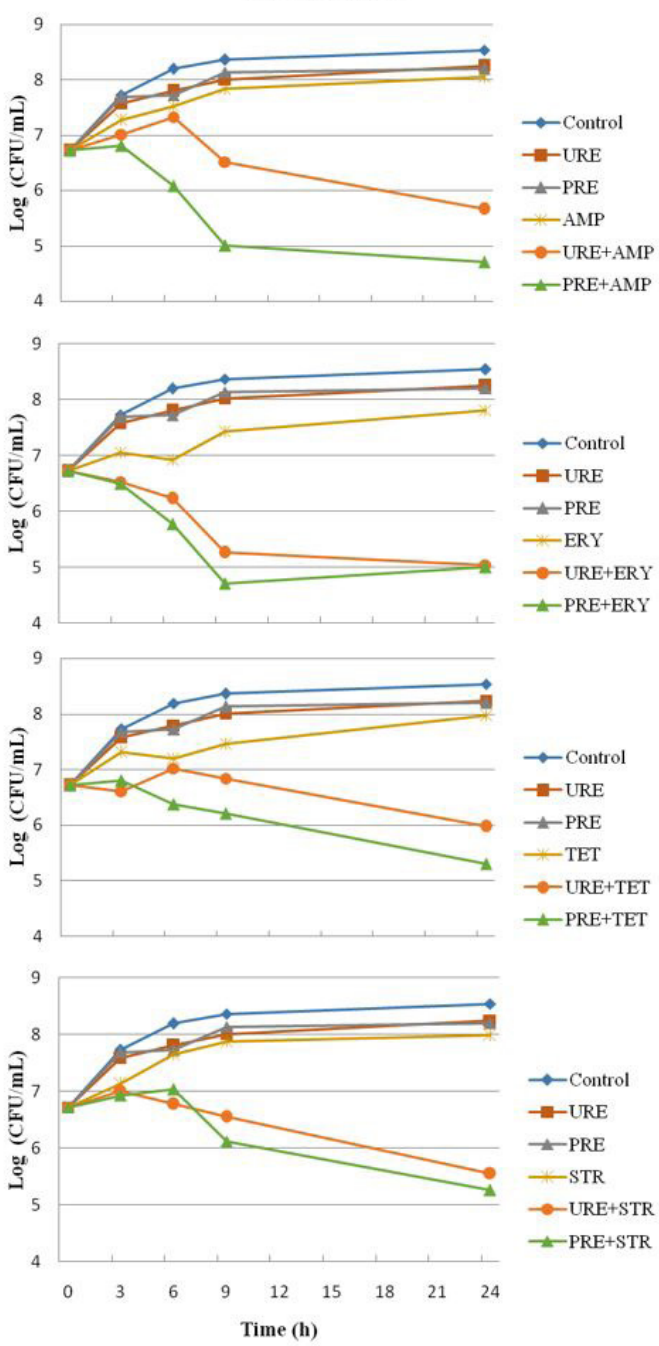

Figure 1. Time kill curves of pairwise combinations of RUE or RPE and antibiotics against bacteria. The experiment was repeated twice with similar results. The data was from one of the two repeated experiments. MIC, minimal inhibitory concentration; CFU, colony forming units; AMP, ampicillin; TET, tetracycline; ERY, erythromycin; STR, streptomycin; URE, unpurified raspberry extract; PRE, purified raspberry extract. Synergy, a decrease of $\geq 2 \times \log _{10} \mathrm{CFU} / \mathrm{mL}$; No interaction, a change of $<2 \times \log _{10} \mathrm{CFU} / \mathrm{mL}$. 
Previous study showed that total phenolic contents of URE and PRE using the Folin-Ciocalteu assay were 233.6 and 357.7 gallic acid $\mathrm{mg} / \mathrm{g}$, respectively. Ellagic acid was the main component of 11 tested polyphenols by HPLC-MS quantitative analysis, constituting $73.00 \%$ in the URE and $67.00 \%$ in the PRE, respectively (Yang et al., 2019). Therefore, we speculated that EA and its derivatives might play important roles in bacteriostatic and synergistic effects because of their high contents in the URE and PRE. For example, EA and EA derivatives from Rubus ulmifolius in particular inhibited S. aureus biofilm formation and improved responses to antibiotics (daptomycin, clindamycin and oxacillin) (Quave et al., 2012). Propionibacterium acnes is a major factor in facial skin infections in adolescents. A combination of EA and TET effectively inhibited the biofilm formation of $P$. acnes without affecting its growth, which potentially limited the possibility of the bacterium attaining resistance (Sivasankar et al., 2016). Moreover, leaf-derived EA from Lagerstroemia speciosa strongly inhibited the RNA replication of HRV-4 in HeLa cells, which was a lead molecule for the prevention of Human rhinovirus infections (Park et al., 2014). The EA content of Euphorbia humifusa may be responsible for treating fungal diseases in Uighur Traditional Medicine. The antifungal activity of EA was slightly lower than that of a commercial antifungal, fluconazole, against Trichophyton rubrum in vivo and in vitro (Li et al., 2015). Han et al. (2016) reported that the $R$. chingii extract combined with fluconazole acted against fluconazole-resistant Candida albicans. Thus, EA and plant-derived EA had antibacterial, antifungal and synergistic activities.

In addition, we speculated that flavonoids of the URE and PRE also participated in bacteriostatic and synergistic effects. For example, kaempferol-3-rutinoside was the main flavonoids of tested polyphenols, constituting $53.42 \%(\sim 0.9 \mathrm{mg} / \mathrm{g} \mathrm{DW})$ in the URE and $46.28 \%(\sim 1.6 \mathrm{mg} / \mathrm{g} \mathrm{DW})$ in the PRE (Yang et al., 2019). It was reported that the apparent synergistic interactions of EA with resveratrol and quercetin that existed in grape, grape extract and grape juice and induce apoptosis in human leukemia cells (Mertens-Talcott \& Percival, 2005). Punicalagin (PU), myricetin (MY), quercetin-3-glucoside (Q3G) and EA were identified as the major flavonoids and polyphenols of a Cistus salviifolius extract by HPLC-ESI-MS/MS, and 26 of 36 pairwise combinations $(\mathrm{Q} 3 \mathrm{G}+\mathrm{MY}, \mathrm{EA}+\mathrm{PU}, \mathrm{Q} 3 \mathrm{G}+\mathrm{PU}, \mathrm{MY}+\mathrm{EA}, \mathrm{MY}+\mathrm{PU}$ and $\mathrm{EA}+\mathrm{Q} 3 \mathrm{G}$ ) at six levels, showed significant synergistic effect against S. aureus growth (Tomas-Menor et al., 2015). Quercetin efficiently inhibited 12 different protein structures and functions (with $\mathrm{IC}_{50}<20 \mu \mathrm{M}$, according to htttp://www.ebi.ac.uk/chembldb/) (Zhang et al., 2010). Therefore, the formulae of these polyphenols and flavonoids in plants may promote their own survival and be sources of natural medicines for humans.

In China, more than 100,000 formulae for botanical mixtures have been continuously used to cure diseases over the past thousand years. In other Asian and African countries, a percentage of the populations depends on traditional medicines as part of their primary health care according to WHO data (World Health Organization, 2010). Even in the UK, a number of cancer patients (from 7\% to $48 \%$ ) report taking botanical medicines after diagnosis (Gratus et al., 2009). A sumac/raspberry mixture is a strong inhibitor of cancer cell proliferation, while having less effect on normal cells (Wang et al., 2015). However, the pharmacological components and synergistic effects of the vast amount of foods and/or herbs have not been clarified even with the development of analytical chemistry and molecular biology. Thus, further research is still needed.

\section{Conclusions}

All pairwise combinations of URE or PRE and antibiotics against bacteria showed synergistic effects $(0.10 \leq \mathrm{FICI} \leq 0.5)$. In particular, the ERY concentration in the combinations was reduced to $1 / 50-1 / 100$ of its normal independent dose while retaining its effectiveness against $S$. aureus and E. coli. EA may play an important role in the synergistic effects, based on the high content of EA in extracts $[73.00 \%(9 \mathrm{mg} / \mathrm{g})$ in the URE and $67.00 \%(12 \mathrm{mg} / \mathrm{g})$ in the PRE]. Understanding the potential of unripe raspberry extract to fight more diseases in vitro or in vivo, will allow a comprehensive exploitation of raspberry extract, which may be a dietary supplement and/or potential medicine.

\section{Acknowledgements}

This research was supported by Opening Project Fund of Key Laboratory of Biology and Genetic Resources of Rubber Tree, Ministry of Agriculture and Rural Affairs, P. R. China/ State Key Laboratory Incubation Base for Cultivation \& Physiology of Tropical Crops / Danzhou Investigation \& Experiment Station of Tropical Crops, Ministry of Agriculture and Rural Affairs, P. R. China (RRI-KLOF201901) and the National Natural Science Foundation of China for Youth (No. 31200462).

\section{References}

Baym, M., Lieberman, T. D., Kelsic, E. D., Chait, R., Gross, R., Yelin, I., \& Kishony, R. (2016a). Spatiotemporal microbial evolution on antibiotic landscapes. Science, 353(6304), 1147-1151. http://dx.doi. org/10.1126/science.aag0822. PMid:27609891.

Baym, M., Stone, L. K., \& Kishony, R. (2016b). Multidrug evolutionary strategies to reverse antibiotic resistance. Science, 351(6268), aad3292. http://dx.doi.org/10.1126/science.aad3292. PMid:26722002.

Belica, A. L., Cetkovic, N. B., Milic, N. B., \& Milosevic, N. P. (2017). Herbal therapy in pregnancy - what to expect when you expect? Natural Product Communications, 12(12), 1957-1969. http://dx.doi. org/10.1177/1934578X1701201236.

Chen, C. Y., Milbury, P. E., Lapsley, K., \& Blumberg, J. B. (2005). Flavonoids from almond skins are bioavailable and act synergistically with vitamins $\mathrm{C}$ and $\mathrm{E}$ to enhance hamster and human LDL resistance to oxidation. The Journal of Nutrition, 135(6), 1366-1373. http:// dx.doi.org/10.1093/jn/135.6.1366. PMid:15930439.

Chinese Pharmacopoeia Commission. (2015). The chinese pharmacopoeia (Vol. 1, 382 p.). China: China Medical Science Press.

Eumkeb, G., \& Chukrathok, S. (2013). Synergistic activity and mechanism of action of ceftazidime and apigenin combination against ceftazidime-resistant Enterobacter cloacae. Phytomedicine, 20(3-4), 262-269. http://dx.doi.org/10.1016/j.phymed.2012.10.008. PMid:23218402.

Fournier-Larente, J., Morin, M. P., \& Grenier, D. (2016). Green tea catechins potentiate the effect of antibiotics and modulate adherence and gene expression in Porphyromonas gingivalis. Archives of Oral 
Biology, 65, 35-43. http://dx.doi.org/10.1016/j.archoralbio.2016.01.014. PMid:26849416.

Gertsch, J. (2011). Botanical drugs, synergy, and network pharmacology: forth and back to intelligent mixtures. Planta Medica, 77(11), 10861098. http://dx.doi.org/10.1055/s-0030-1270904. PMid:21412698.

Gillings, M. R. (2013). Evolutionary consequences of antibiotic use for the resistome, mobilome and microbial pangenome. Frontiers in Microbiology, 4(4), 1-10. http://dx.doi.org/10.3389/fmicb.2013.00004. PMid:23386843.

Gratus, C., Wilson, S., Greenfield, S. M., Damery, S. L., Warmington, S. A., Grieve, R., Steven, N. M., \& Routledge, P. (2009). The use of herbal medicines by people with cancer: a qualitative study. BMC Complementary and Alternative Medicine, 9(14), 1-7. http://dx.doi. org/10.1186/1472-6882-9-14. PMid:19442268.

Hall, M. J., Middleton, R. F., \& Westmacott, D. (1983). The fractional inhibitory concentration (FIC) index as a measure of synergy. The Journal of Antimicrobial Chemotherapy, 11(5), 427-433. http://dx.doi. org/10.1093/jac/11.5.427. PMid:6874629.

Han, B., Chen, J., Yu, Y. Q., Cao, Y. B., \& Jiang, Y. Y. (2016). Antifungal activity of Rubus chingii extract combined with fluconazole against fluconazole-resistant Candida albicans. Microbiology and Immunology, 60(2), 82-92. http://dx.doi.org/10.1111/1348-0421.12357. PMid:26891940.

Krauze-Baranowska, M., Majdan, M., Halasa, R., Glod, D., Kula, M., Fecka, I., \& Orzeł, A. (2014). The antimicrobial activity of fruits from some cultivar varieties of Rubus idaeus and Rubus occidentalis. Food \& Function, 5(10), 2536-2541. http://dx.doi.org/10.1039/ C4FO00129J. PMid:25131001.

Li, Z. J., Guo, X., Dawuti, G., \& Aibai, S. (2015). Antifungal activity of ellagic acid in vitro and in vivo. Phytotherapy Research, 29(7), 1019-1025. http://dx.doi.org/10.1002/ptr.5340. PMid:25919446.

Mertens-Talcott, S. U., \& Percival, S. S. (2005). Ellagic acid and quercetin interact synergistically with resveratrol in the induction of apoptosis and cause transient cell cycle arrest in human leukemia cells. Cancer Letters, 218(2), 141-151. http://dx.doi.org/10.1016/j. canlet.2004.06.007. PMid:15670891.

National Committee for Clinical Laboratory Standards - NCCLS. (1999). Methods for determini bactericidal activity of antimicrobial agentsagents (Tentative Guidline, M26-A). Pensylvania, USA: CLSI.

Newman, D. J., \& Cragg, G. M. (2007). Natural products as sources of new drugs over the last 25 years. Journal of Natural Products, 70(3), 461-477. http://dx.doi.org/10.1021/np068054v. PMid:17309302.

Odds, F. C. (2003). Synergy, antagonism, and what the chequerboard puts between them. The Journal of Antimicrobial Chemotherapy, 52(1), 1. http://dx.doi.org/10.1093/jac/dkg301. PMid:12805255.

Park, S. W., Kwon, M. J., Yoo, J. Y., Choi, H. J., \& Ahn, Y. J. (2014). Antiviral activity and possible mode of action of ellagic acid identified in Lagerstroemia speciosa leaves toward human rhinoviruses. BMC Complementary and Alternative Medicine, 14(17), 1-8. http://dx.doi. org/10.1186/1472-6882-14-171. PMid:24885569.

Popowska, M., Rzeczycka, M., Miernik, A., Krawczyk-Balska, A., Walsh, F., \& Duffy, B. (2012). Influence of soil use on prevalence of tetracycline, streptomycin, and erythromycin resistance and associated resistance genes. Antimicrobial Agents and Chemotherapy, 56(3), 1434-1443. http://dx.doi.org/10.1128/AAC.05766-11. PMid:22203596.

Quave, C. L., Estevez-Carmona, M., Compadre, C. M., Hobby, G., Hendrickson, H., Beenken, K. E., \& Smeltzer, M. S. (2012). Ellagic acid derivatives from Rubus ulmifolius inhibit Staphylococcus aureus biofilm formation and improve response to antibiotics. PLoS One, 7(1), e28737. http://dx.doi.org/10.1371/journal.pone.0028737. PMid:22242149.
Schmidt, B. M., Ribnicky, D. M., Lipsky, P. E., \& Raskin, I. (2007). Revisiting the ancient concept of botanical therapeutics. Nature Chemical Biology, 3(7), 360-366. http://dx.doi.org/10.1038/ nchembio0707-360. PMid:17576417.

Sivasankar, C., Maruthupandiyan, S., Balamurugan, K., Bhaskar, J. P., Krishnan, V., \& Pandian, S. K. (2016). A combination of ellagic acid and tetracycline inhibits biofilm formation and the associated virulence of Propionibacterium acnes in vitro and in vivo. Biofouling, 32(4), 397-410. http://dx.doi.org/10.1080/08927014.2016.1148141 . PMid:26930280.

Tomas-Menor, L., Barrajon-Catalan, E., Segura-Carretero, A., Marti, N., Saura, D., Menendez, J. A., Joven, J., \& Micol, V. (2015). The promiscuous and synergic molecular interaction of polyphenols in bactericidal activity: an opportunity to improve the performance of antibiotics? Phytotherapy Research, 29(3), 466-473. http://dx.doi. org/10.1002/ptr.5296. PMid:25625775.

Wang, L., Zhou, G. B., Liu, P., Song, J. H., Liang, Y., Yan, X. J., Xu, F., Wang, B. S., Mao, J. H., Shen, Z. X., Chen, S. J., \& Chen, Z. (2008). Dissection of mechanisms of Chinese medicinal formula RealgarIndigo naturalis as an effective treatment for promyelocytic leukemia. Proceedings of the National Academy of Sciences of the United States of America, 105(12), 4826-4831. http://dx.doi.org/10.1073/ pnas.0712365105. PMid:18344322.

Wang, S. N., Meckling, K. A., Marcone, M. F., Kakuda, Y., Proulx, A., \& Tsao, R. (2012). In vitro antioxidant synergism and antagonism between food extracts can lead to similar activities in $\mathrm{H}_{2} \mathrm{O}_{2}$-induced cell death, caspase- 3 and MMP-2 activities in H9c2 cells. Journal of the Science of Food and Agriculture, 92(15), 2983-2993. http:// dx.doi.org/10.1002/jsfa.5711. PMid:22538730.

Wang, S. N., Zhu, F., \& Marcone, M. F. (2015). Synergistic Interaction of sumac and raspberry mixtures in their antioxidant capacities and selective cytotoxicity against cancerous Cells. Journal of Medicinal Food, 18(3), 345-353. http://dx.doi.org/10.1089/jmf.2013.0171. PMid:25313437.

Wei, C. F., Chang, S. K., Shien, J. H., Kuo, H. C., Chen, W. Y., \& Chou, C. C. (2016). Synergism between two amphenicol of antibiotics, florfenicol and thiamphenicol, against Staphylococcus aureus. The Veterinary Record, 178(13), 1-6. http://dx.doi.org/10.1136/vr.103554. PMid:26864028.

World Health Organization - WHO. (2010). Traditional medicine. Geneva: WHO.

Xiao, T., Guo, Z. H., Bi, X. L., \& Zhao, Y. Q. (2017). Polyphenolic profile as well as anti-oxidant and anti-diabetes effects of extracts from freeze-dried black raspberries. Journal of Functional Foods, 31, 179-187. http://dx.doi.org/10.1016/j.jff.2017.01.038.

Yang, J., Cui, J. Y., Wu, Y. H., Han, H. J., Chen, J. X., Yao, J. Y., \& Liu, Y. (2019). Comparisons of the active components in four unripe raspberry extracts and their activites. Food Science and Technology (Campinas), 39(suppl 2upl. suppl 2), 632-639. http://dx.doi. org/10.1590/fst.27418.

Yang, Y., Zhang, Z., Li, S., Ye, X., Li, X., \& He, K. (2014). Synergy effects of herb extracts: pharmacokinetics and pharmacodynamic basis. Fitoterapia, 92, 133-147. http://dx.doi.org/10.1016/j.fitote.2013.10.010. PMid:24177191.

Yu, G., Luo, Z., Wang, W., Li, Y., Zhou, Y., \& Shi, Y. (2019). Rubus chingii $\mathrm{Hu}$ : a review of the phytochemistry and pharmacology. Frontiers in Pharmacology, 10, 1-22. http://dx.doi.org/10.3389/fphar.2019.00799. PMid:31379574.

Zhang, H. Y., Chen, L. L., Li, X. J., \& Zhang, J. (2010). Evolutionary inspirations for drug discovery. Trends in Pharmacological Sciences, 31(10), 443-448. http://dx.doi.org/10.1016/j.tips.2010.07.003. PMid:20724009. 


\section{Supplementary Material}

Supplementary material accompanies this paper.

Table S1. Inhibitory activity of raspberry extract and antibiotics alone and in combination against bacteria.

This material is available as part of the online article from 